\title{
Interferon and meta-iodobenzylguanidin combinations in the treatment of metastatic carcinoid tumours
}

\author{
$J$ M Zuetenhorst, $R$ A Valdes Olmos ${ }^{1}, M$ Muller $^{2}, C$ A Hoefnagel ${ }^{1}$ \\ and $B$ G Taal
}

\author{
Department of Gastroenterology, Netherlands Cancer Institute/Antoni van Leeuwenhoek Hospital, Plesmanlaan \\ 121, 1066 CX Amsterdam, The Netherlands \\ ${ }^{1}$ Department of Nuclear Medicine, Netherlands Cancer Institute/Antoni van Leeuwenhoek Hospital, \\ Amsterdam, The Netherlands \\ ${ }^{2}$ Department of Psychology, Netherlands Cancer Institute/Antoni van Leeuwenhoek Hospital, Amsterdam, \\ The Netherlands
}

(Requests for offprints should be addressed to J M Zuetenhorst; Email: j.zuetenhorst@nki.nl)

\begin{abstract}
Interferon (IFN) and meta-iodobenzylguanidin (MIBG) are active in metastatic carcinoids. In a phase II study, we evaluated the effect upon diagnostic ${ }^{131}$ I-MIBG uptake and the clinical response of the combination.

${ }^{131}$ I-MIBG scintigraphy was performed prior to treatment, after 8 weeks of IFN and after unlabelled MIBG. The tumour over non-tumour (T/NT) ratios were quantitatively determined by comparing counts in the centre of the tumour (liver metastases) with those in an adjacent area of normal liver uptake (T/NT1) and with abdominal background area (T/NT2).

The T/NT1 ratio showed an increase of $>10 \%$ in only four out of 21 patients $(19 \%)$ after IFN $(P=0.178)$ and significantly more often in nine out of 18 patients $(50 \%)$ after unlabelled MIBG $(P=0.016)$. The absolute uptake in tumour deposits was also increased if compared with the abdominal background (T/NT2: 23\% increase after IFN and 83\% increase after unlabelled MIBG). The combination produced $91 \%$ of patients with stable disease (using World Health Organisation criteria) at computed tomography scan and a biochemical response (a reduction of at least $50 \%$ in urinary 5-hydroxyindolacetic acid excretion) in 39\%.

IFN- $\alpha$ did not significantly improve tumour retention of ${ }^{131}$ I-MIBG. In contrast, unlabelled MIBG significantly improved biodistribution and tumour uptake in $83 \%$. A synergistic effect was not seen.
\end{abstract}

Endocrine-Related Cancer (2004) 11 553-561

\section{Introduction}

Carcinoid tumours are neuroendocrine tumours associated with the synthesis and secretion of biologically active substances that can induce characteristic symptoms of the carcinoid syndrome (flushing, diarrhoea and wheezing). Metastatic disease at diagnosis is found in $22 \%$, and is largely dependent on the primary localisation: appendix $1.5 \%$, lung $4.8 \%$ and small and large bowel localisations 28\% (excluding appendix and rectum) (Quaedvlieg et al. 2001). Various treatment strategies have been recommended, mainly aimed at amelioration of the incapacitating symptoms (Moertel et al. 1989, Oberg 1994). Surgery is indicated for the primary tumour in cases of obstruction.
In metastatic disease of the liver, palliative resection may be of value, but is usually not feasible because of diffuse involvement of both liver lobes. For medical treatment, somatostatin analogues and/or interferon (IFN)- $\alpha$ formulations are employed by many clinicians, as biochemical and subjective responses are observed in $70 \%$ and $80 \%$ respectively for somatostatin analogues (Harris \& Redfern 1995) and 40\% and 70\% respectively for IFN- $\alpha$ formulations (Oberg et al. 1994). Pharmacological doses of meta-iodobenzylguanidin (MIBG) resulted in symptomatic improvement in $60 \%$ of carcinoid patients (Taal et al. 1996). The cytotoxic effect of MIBG is related to the inhibition of mitochondrial respiration and is dependent on anaerobic glycolysis, resulting in enhanced glucose 
consumption, increased lactic acid production, inhibition of oxygen consumption and decreased adenosine triphosphate levels (Kuin et al. 1994). Another possibility is treatment with radiolabelled MIBG, based on a positive scintigraphy which leads to long-term palliation in $60 \%$ of patients. Although reduction in tumour growth is rarely reported, stable disease is described in a substantial proportion of the patients (Taal et al. 1996, 2000, Caplin et al. 1998, Mukherjee et al. 2001, Pathirana et al. 2001). Worldwide experience with ${ }^{131}$ I-MIBG diagnostic imaging has indicated a cumulative sensitivity of $70 \%$ in 237 patients with carcinoid tumours (Hoefnagel 1991). Predosing with unlabelled MIBG resulted in improved tumour targeting, biochemical response and hence prolonged palliation (Zuetenhorst et al. 1999, Taal et al. 2000). Carcinoids are known to take up amino acid precursors for the endogenous production of serotonin. Recent reports suggest a role of vesicular monoamine transporters in this uptake (Eissele et al. 1999, Kolby et al. 2003). Pretreatment with unlabelled MIBG can produce a saturation of normal tissues with a preferential binding of the radioactive labelled MIBG to carcinoid tumour cells. Another option to increase MIBG tumour targeting might be pretreatment with INF- $\alpha$. This assumption is based on the laboratory findings of an improved uptake of ${ }^{125}$ I-MIBG following IFN- $\gamma$ administration in neuroblastoma cell lines. This phenomenon was based on de novo synthesis of MIBG receptor transporters (Montaldo et al. 1992). Although INF- $\alpha$ is different from INF- $\gamma$, differentiation of carcinoid tumour cells with the application of IFN- $\alpha$ has been described, with good clinical results (Oberg 1992). It was hypothesised that the combination of two active treatment modalities (IFN and MIBG) may lead to an additive or even synergistic effect.

In the currently presented phase II study, we examined the effect of IFN- $\alpha$ upon diagnostic ${ }^{131}$ I-MIBG uptake by carcinoid tumours, and whether the clinical response, including quality of life, to IFN- $\alpha$ therapy was improved by MIBG. In addition IFN- $\alpha$ antibody formation was evaluated for its influence on the results of the therapy.

\section{Patients and methods Patients}

Patients who were referred to our hospital between October 1996 and December 1998 with metastatic carcinoid tumours, a World Health Organisation (WHO) performance status of 0-2 and normal blood cell counts and renal function were eligible. Twenty-six consecutive patients (18 men and eight women) entered the study (Table 1). All patients had metastatic, welldifferentiated neuroendocrine tumours, originating from the distal ileum-coecum $(n=6)$, the lungs $(n=5)$, the stomach $(n=1)$ or with an unknown primary site $(n=14)$. Median age was 63 years (range: 41-77). In 23 patients, the diagnosis of neuroendocrine tumour was confirmed by histology. In two patients, fine needle aspiration led to the diagnosis; one patient refused a biopsy and the diagnosis was based on strongly elevated urinary 5-hydoxyindolacetic acid (5-HIAA) excretion and symptoms. Three patients had normal urinary 5-HIAA excretions; median pretreatment urinary 5-HIAA excretion in the 23 secretors was $503 \mu \mathrm{mol} / 24 \mathrm{~h}$ (normal $<40 \mu \mathrm{mol} / 24 \mathrm{~h})$. Twenty-two patients $(85 \%)$ suffered from the carcinoid syndrome with diarrhoea and/or flushes at the time of enrolment. Fifteen patients had complaints related to tumour size or localisation. Median duration of symptoms before the start of treatment was 7 months (range: 0-94 months). In 11 patients, the indication for enrolment was progression under systemic therapy and in two after prior surgery; the remaining patients were not pretreated. Four patients received prior treatment with unlabelled as well as ${ }^{131} \mathrm{I}-\mathrm{MIBG}$; the interval was at least 2.5 years. The study was approved by the local medical ethical committee and written informed consent was given by all patients.

\section{Treatment}

Treatment started with IFN- $\alpha$ 2a (Roferon; Hoffmann-La Roche, Basel, Switzerland), 6 million IU thrice weekly s.c. After 8 weeks, unlabelled MIBG was added in three cycles of increasing doses $\left(10,20\right.$ and $\left.40 \mathrm{mg} / \mathrm{m}^{2}\right)$ in a 4-h infusion every 4 weeks as described earlier (Taal et al. 1996). Subsequently, patients with a positive ${ }^{131} \mathrm{I}-\mathrm{MIBG}$ scan received two therapeutic doses of $7.4 \mathrm{GBq}{ }^{131} \mathrm{I}-\mathrm{MIBG}$ administered during a hospital stay (Taal et al. 1996). IFN- $\alpha 2$ a therapy was continued for 6 months and only stopped if serious side-effects persisted or in cases of progressive disease. During this study, ten patients were treated with somatostatin analogues which were continued in a stable dosage.

\section{Methods}

The effect of IFN- $\alpha$ upon ${ }^{131}$ I-MIBG tumour uptake was evaluated by comparing ${ }^{131}$ I-MIBG scans prior to and after 8 weeks of treatment. Subsequently, the effect of unlabelled MIBG was evaluated by the ${ }^{131}$ I-MIBG scan directly following the infusion of unlabelled MIBG. The tumour over non-tumour (T/NT) ratio was quantitatively determined by comparing counts in the centre of the tumour (liver metastases) with those in an adjacent area of normal liver uptake (T/NT1) and with abdominal background area (T/NT2) as described earlier (Hoefnagel et al. 2000). In cases of extrahepatic tumour localisation, the 
Table 1 Clinical characteristics of the patients

\begin{tabular}{|c|c|}
\hline Total group of patients & 26 \\
\hline \multicolumn{2}{|l|}{ Sex } \\
\hline Male & 18 \\
\hline Female & 8 \\
\hline \multicolumn{2}{|l|}{ Age (years) } \\
\hline Median (range) & $63(41-77)$ \\
\hline \multicolumn{2}{|l|}{ Primary site } \\
\hline Distal ileum-coecum & 6 \\
\hline Lung & 5 \\
\hline Stomach & 1 \\
\hline Unknown & 14 \\
\hline \multicolumn{2}{|l|}{ Therapy (no. of patients) } \\
\hline None & 14 \\
\hline Somatostatin analogues & 10 \\
\hline IFN- $\alpha 2 a$ & 0 \\
\hline MIBG (> 2.5 years before study) & 4 \\
\hline${ }^{131}$ I-MIBG (> 2.5 years before study) & 5 \\
\hline Chemotherapy & 2 \\
\hline Radiotherapy (skin metastases) & 1 \\
\hline \multicolumn{2}{|l|}{ Duration of symptoms before start of study (months) } \\
\hline Median (range) & $7(0-94)$ \\
\hline \multicolumn{2}{|l|}{ Predominant symptoms } \\
\hline Fever/weight loss/sweating/fatigue & 8 \\
\hline Carcinoid syndrome & 14 \\
\hline Abdominal pain (unrelated to carcinoid syndrome) & 3 \\
\hline None & 1 \\
\hline \multicolumn{2}{|l|}{ Indication for starting the protocol } \\
\hline First-line therapy & 13 \\
\hline Progression under therapy & 13 \\
\hline \multicolumn{2}{|l|}{ Urinary 5 -HIAA excretion $(\mu \mathrm{mol} / 24 \mathrm{~h})$ at start of study } \\
\hline$<$ ULN $(n=3)$ median (range) & $23(21-34)$ \\
\hline$>$ ULN $(n=23)$ median (range) & $503(130-3131)$ \\
\hline
\end{tabular}

5-HIAA, normal $<40 \mu \mathrm{mol} / 24 \mathrm{~h}$; ULN, upper limit of normal.

ratio was calculated by comparing this particular localisation with the abdominal background (T/NT3). A 10\% increase in biodistribution was considered a clinical important difference.

Computed tomography (CT) scans were performed before the start, after 8 weeks of IFN treatment and after the third course of unlabelled MIBG and after the last course of ${ }^{131} \mathrm{I}-\mathrm{MIBG}$. CT scans were systematically reviewed without clinical information to classify the liver metastases into two categories: nodular and diffuse. Objective responses by tumour size were assessed using the WHO criteria. Complete response: disappearance of all target lesions; partial response (PR): $>50 \%$ decrease of bi-dimensionally measured diameters; progressive disease (PD): $>25 \%$ increase in size of measurable lesions or appearance of a new lesion; stable disease (SD): neither PD nor PR. A biochemical response was defined as a reduction of at least $50 \%$ in urinary 5-HIAA excretion. Adverse effects were scored using the common toxicity criteria.

IFN- $\alpha 2$ antibody concentrations before and after at least 4 months of IFN therapy were analysed using an enzyme immunoassay method (Schellekens et al. 1997). An antibody titre rise was considered significant if the post-IFN value exceeded the 95th percentile of the groups' pre-IFN values, and the rise was at least $50 \%$ of the pre-IFN value.

The effect on quality of life was measured with the EORTC quality of life questionnaire QLQ-C30 (Wymenga et al. 1999, Langendijk et al. 2001). Questions were added to evaluate the specific carcinoid symptoms, such as flushes and abdominal cramps, and the specific sideeffects of IFN such as chills and muscle pain. All scores were linearly converted to a 0-100 scale. For the functional and global health status/quality of life scales, higher scores represent a better level of functioning. For the symptom scales, higher scores represent a greater degree of symptoms. The Center for Epidemiologic Studies depression (CES-D) scale list of 20 items was included to measure depression (Radloff 1977). A score of 16 or more indicated depression (Geerlings et al. 2000, Sarna et al. 2002). Finally, the symptoms as noted by the physician in the medical record were evaluated. According 
to these findings, patients were divided into four groups based on the predominant symptoms: (A) patients with the characteristic carcinoid syndrome of flushing, diarrhoea and/or wheezing; (B) patients with systemic symptoms such as fever, anorexia, sweating and/or fatigue; (C) patients with abdominal pain; (D) patients without symptoms. These main symptoms were considered to be improved if the patient experienced a clear reduction (e.g. weight gain or $>50 \%$ less additional medication) or disappearance of symptoms, and progression in cases of deterioration. Survival and time to progression (TTP) were measured from the start of treatment. TTP was defined as the number of months between start of IFN and a change in therapeutic regimen because of progression.

For statistical analysis, the two-sided, paired $t$-test was applied with a $P$ value of 0.05 as the level of significance. Survival curves were constructed using the Kaplan-Meier method and compared using a log-rank test. Categorical differences between groups were compared using Fishers' exact test. For all calculations SPSS 10.0 (SPSS Inc., Chicago, USA) was used.

\section{Results}

\section{Protocol evaluation}

Twenty-three patients completed the first 8 weeks of IFN therapy. Three patients stopped within 8 weeks because of clinical progression.

Twenty patients were able to complete the full-course of three MIBG cycles and reached follow-up at 21 weeks. Two patients were progressive between the first and the second treatment with MIBG. In one patient, the third treatment with MIBG was not administered as the previous two failed to achieve relief of symptoms.

Among the twenty patients who reached week 21, seven patients received two cycles of $7.4 \mathrm{GBq}{ }^{131} \mathrm{I}-\mathrm{MIBG}$ and fully completed the protocol. Seven patients received only one cycle of ${ }^{131}$ I-MIBG: two patients because of progressive disease, two patients based on thrombocytopenia, one patient pretreated with ${ }^{131}$ I-MIBG reached his cumulative maximal radiation dose, and in two patients a temporary drop in WHO performance status prevented further ${ }^{131}$ I-MIBG infusion. Six patients were not given
${ }^{131}$ I-MIBG as their ${ }^{131}$ I-MIBG scan was negative, but they continued IFN- $\alpha$ as planned.

\section{Responses, TTP, survival and adverse effects}

In 22 patients $(85 \%)$ the tumour lesions remained stable at evaluation of the CT scan after 8 weeks of treatment with IFN, in one there was PD. In addition, three patients were rapidly progressive in terms of symptoms and 5-HIAA excretion and a follow-up CT scan was not performed. In one patient with no change at prior chemotherapy, the primary tumour showed PR, while the liver metastases remained stable. At 21 weeks, after three courses of unlabelled MIBG, two additional patients were clinically progressive and no follow-up CT scans were performed. The remaining 20 patients $(91 \%)$ showed SD. At 34 weeks, after two courses of ${ }^{131} \mathrm{I}-\mathrm{MIBG}$, two out of 20 patients were clinically progressive and a follow-up CT scan was not available. One had PD and 17 had SD. Therefore, none of the patients had a PR, defined by CT criteria and, after 34 weeks, 17 out of $26(65 \%)$ patients had SD.

After 8 weeks of IFN therapy and using biochemical evaluation of urinary 5-HIAA excretion, PR values were reached in eight out of $23(35 \%)$ patients with elevated urinary 5-HIAA. At 21 weeks, one additional patient who had previously shown SD reached PR. The protocol resulted in an overall best response rate (according to 5 -HIAA excretion) of $39 \%$.

The $\mathrm{T} / \mathrm{NT} 1$ ratio of the ${ }^{131} \mathrm{I}-\mathrm{MIBG}$ retention showed a $10 \%$ increase in only four out of 21 patients $(19 \%)$ after IFN treatment, while this was the case in nine out of 18 patients $(50 \%)$ after unlabelled MIBG (Table 2). Using the $\mathrm{T} / \mathrm{NT} 2$ ratio, these figures were even more pronounced: $23 \%$ after IFN and $83 \%$ after unlabelled MIBG (Fig. 1). This improved biodistribution was not merely based on a decrease in background activity in the liver, but the absolute uptake in tumour deposits was increased if compared with the abdominal background. Extra-hepatic tumour localisation was present in only seven patients and the T/NT3 ratio showed a similar effect after IFN and MIBG with $14 \%$ and $83 \%$ respectively. Overall, the changes after IFN treatment were not statistically sig-

Table 2 Changes in ${ }^{131}$ I-MIBG retention after IFN treatment and application of unlabelled MIBG

\begin{tabular}{lllllc}
\hline & \multicolumn{2}{c}{ T/NT1 ratio } & & \multicolumn{2}{c}{ T/NT2 ratio } \\
\cline { 2 - 3 } \cline { 6 - 6 } & \multicolumn{1}{c}{ After IFN } & After MIBG & & After IFN & After MIBG \\
\hline$>10 \%$ increase in ${ }^{131}$ I-MIBG uptake & $4 / 21(19 \%)$ & $9 / 18(50 \%)$ & & $5 / 21(23 \%)$ & $15 / 18(83 \%)$ \\
No change in ${ }^{131}$ I-MIBG uptake & $16 / 21(76 \%)$ & $7 / 18(39 \%)$ & & $10 / 21(48 \%)$ & $3 / 18(17 \%)$ \\
$>10 \%$ decrease in ${ }^{131}$ I-MIBG uptake & $1 / 21(5 \%)$ & $2 / 18(11 \%)$ & & $6 / 21(29 \%)$ & $0 / 18(0 \%)$ \\
\hline
\end{tabular}



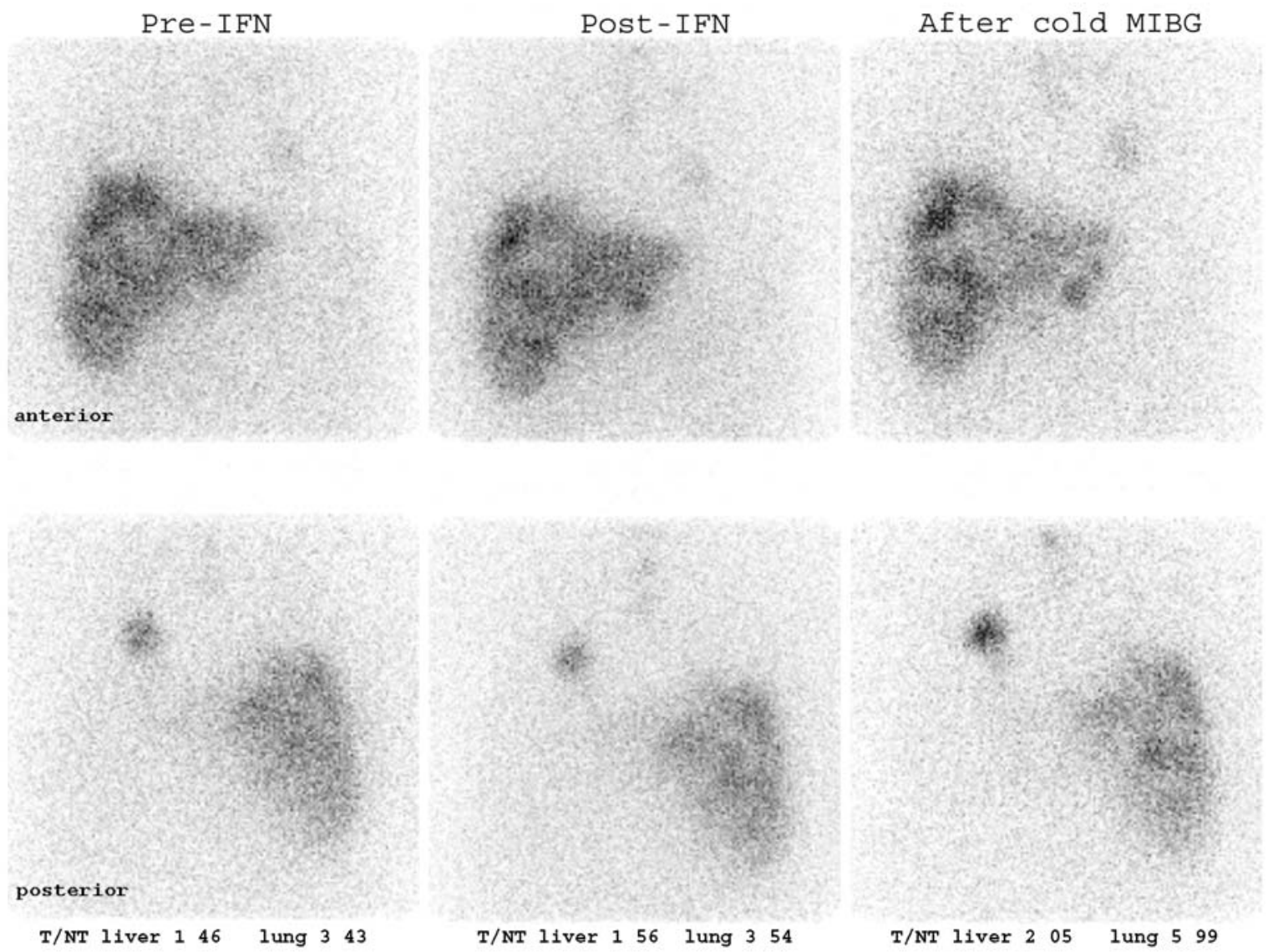

Figure $1{ }^{131}$ I-MIBG scintigraphy before (pre-IFN) and after (post-IFN) treatment with IFN and after treatment with unlabelled MIBG (after cold MIBG). Uptake in liver metastases compared with normal liver tissue (T/NT1) and lung metastases compared with abdominal background (T/NT3) shows a minimal increase after IFN of $7 \%$ and $3 \%$ respectively. After application of unlabelled MIGB, uptake in both localisations was increased by $31 \%$ and $69 \%$ respectively. The quantitatively determined uptake by comparing counts in the centre of the tumour is represented below the scintigrams.

nificant $(P=0.178)$. In contrast, a significant $(P=0.016)$ increase after treatment with unlabelled MIBG was found.

The median TTP (Fig. 2) was 9 months and after 18 months $90 \%$ of the patients had PD. Median overall survival (Fig. 2) from the start of the present treatment protocol was 27 months, while the 5-year survival was $33 \%$. In patients with an increased tumour retention at ${ }^{131}$ I-MIBG scintigraphy after IFN, survival was comparable $(P=0.89)$ with the others (median 32 versus 38 months). Nor was there a difference in survival $(P=0.80)$ for patients with increased tumour retention induced by unlabelled MIBG $(n=9)$. The presence of diffuse versus nodular liver metastases did not result in a difference in survival. However, in pretreated patients, survival tended $(P=0.20)$ to be shorter compared with patients who were not pretreated (18 versus 37 months).

IFN-related toxicity was mild and transient: nine patients suffered from transient influenza-like symptoms and eight from fatigue. Severe toxicity was seen in one patient suffering from grade III hypertension during unlabelled MIBG infusion and in one patient suffering from grade III thrombocytopenia after ${ }^{131}$ I-MIBG infusion. One patient developed a carcinoid crisis following ${ }^{131}$ I-MIBG infusion. All side-effects were reversible and there were no toxic deaths.

\section{Antibodies against recombinant human IFN- $\alpha$ $2 a$ and TTP}

A second blood sample used for IFN- $\alpha$ antibody titre determination, taken 4-6 months after the start of the IFN treatment, was available in 19 patients with a median time of IFN treatment of 6.5 months (range: 4.1-9.2). Six of these 19 patients $(32 \%)$ developed a rise in antibody titre. Patient characteristics, including gender, age, primary site, previous therapies, urinary 5-HIAA values and disease 


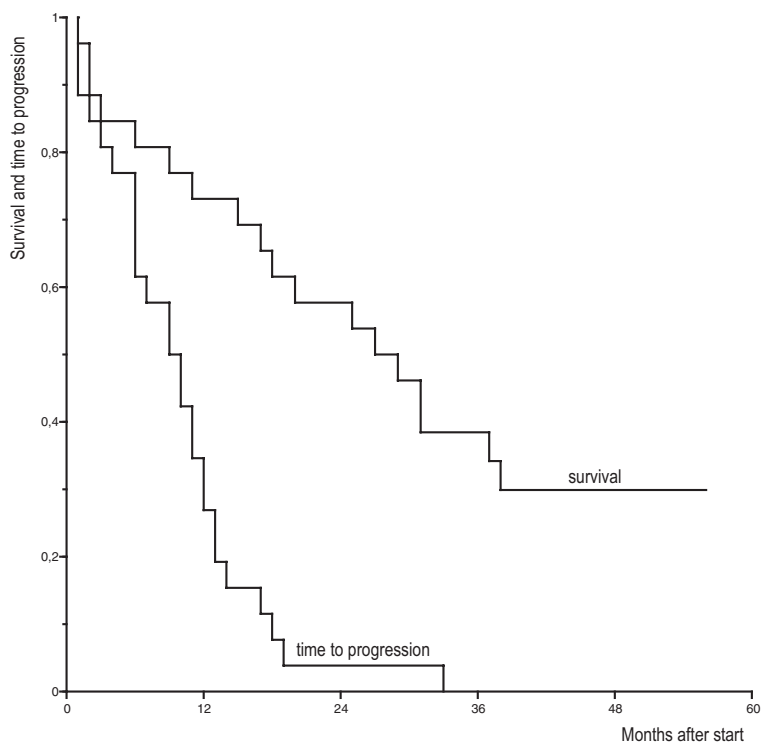

Figure 2 Survival and TTP. Median TTP was 9 months, median survival was 27 months with a 5 -year survival of $33 \%$. Eight patients were still alive during the last follow-up.

duration at the start of the protocol were not significantly different between patients with and without development of antibodies. A difference in response and TTP was not found, but overall survival (Fig. 3) was significantly better in patients without antibody formation $(P=0.04)$.

\section{Symptoms, quality of life and depression}

As summarised in Table 3, quality of life measurement at week 8 with IFN monotherapy did not show significant changes in the five functioning scales. Among the additional single symptoms, flushes and worries about disease progression diminished statistically significant. At week 21, with unlabelled MIBG added to the IFN, appetite deteriorated gradually over time, but this difference was not significant $(P=0.083)$. Abdominal pain increased in the first 8 weeks and then returned to baseline in the subsequent weeks of MIBG treatment.

By comparing patients with predominantly carcinoid symptoms $(n=14)$ with patients complaining of other systemic symptoms, such as fever, severe anorexia and sweating $(n=8)$, tumour complaints showed differences in response on IFN and MIBG treatment. Half of the patients $(50 \%)$ with carcinoid syndrome reported improvement after 8 weeks of IFN compared with four out of $14(29 \%)$ following unlabelled MIBG $(P=0.3)$. Similarly, three out of eight $(38 \%)$ patients with systemic symptoms mentioned improvement with IFN, but they did better after unlabelled MIBG with a response in six out of eight $(75 \%)(P=0.14)$. Overall, after MIBG treatment, patients with systemic symptoms responded

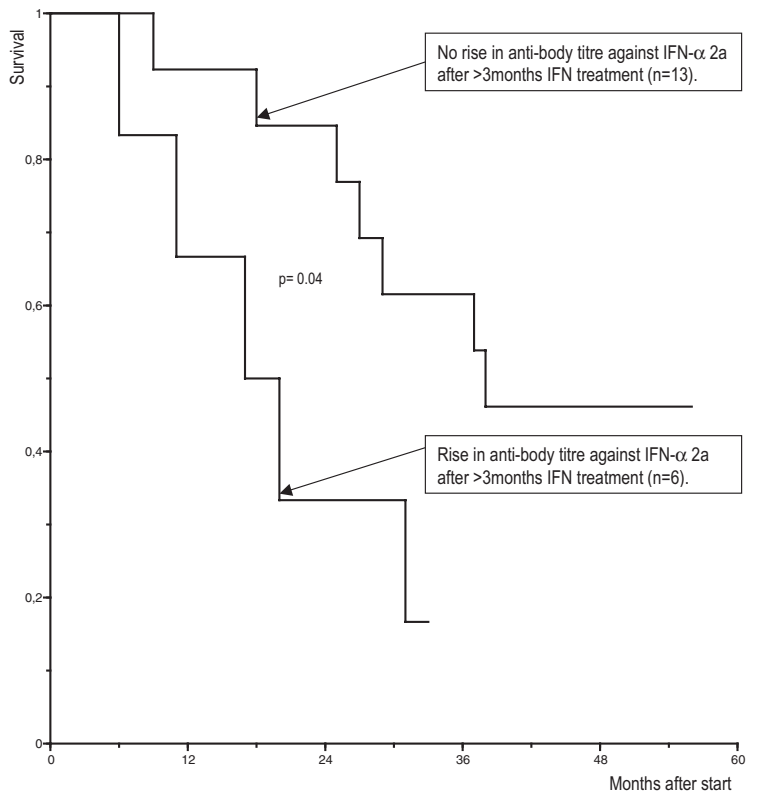

Figure 3 TTP according to the development of an IFN- $\alpha$ 2a titre rise after at least 3 months of IFN- $\alpha 2$ a treatment. In six out of 19 patients $(32 \%)$, a rise in antibodies was observed. Difference in response and TTP were not found. Overall survival was significantly better in patients without antibody formation $(P=0.04)$.

significantly better on MIBG treatment compared with patients with carcinoid syndrome $(P=0.04)$.

\section{Discussion}

IFN- $\alpha$ formulations are well known for their therapeutic effect in metastatic carcinoid disease: subjective responses can be achieved in up to $70 \%$ of patients and biochemical responses are observed in 40-50\% (Moertel et al. 1989, Oberg et al. 1994). A measurable tumour reduction was, however, only occasionally reported. The anti-proliferative effect of IFN- $\alpha$ has, at least in part, been ascribed to a delay in the $\mathrm{S}-\mathrm{G}_{2} / \mathrm{M}$ phase transit (Detjen et al. 2000). In agreement with the literature, the present study showed a biochemical response rate of $35 \%$ with $\operatorname{IFN}-\alpha \quad 2 \mathrm{a}$ monotherapy, but in one patient the primary tumour showed a PR, while the liver metastases remained stable. Side-effects were mild and limited to influenza-like symptoms and sometimes fatigue, both responded well to paracetamol.

In $32 \%$ of our patients, neutralising antibodies were detected during follow-up. The response to the treatment protocol and the TTP were not different, although the overall survival was worse in the presence of antibodies. However, the role of these antibodies is controversial as the presence of these antibodies is probably not related to the response to IFN administration, at least in patients 
Table 3 Quality of life by EORTC QLQ-C30 and depression score by CES-D

\begin{tabular}{|c|c|c|c|c|c|c|c|c|c|c|c|c|}
\hline & \multirow[b]{2}{*}{$n$} & \multirow[b]{2}{*}{$\begin{array}{l}\text { Baseline } \\
\text { mean }\end{array}$} & \multirow[b]{2}{*}{ S.D. } & \multicolumn{3}{|c|}{$\begin{array}{c}\text { Mean change } \\
\mathrm{T}_{0}-\mathrm{T}_{8}\end{array}$} & \multirow[b]{2}{*}{$n$} & \multirow[b]{2}{*}{$\begin{array}{l}\text { Week } 8 \\
\text { mean }\end{array}$} & \multirow[b]{2}{*}{ S.D. } & \multicolumn{3}{|c|}{$\begin{array}{l}\text { Mean change } \\
\qquad T_{8}-T_{21}\end{array}$} \\
\hline & & & & Mean & S.D. & $P$ & & & & Mean & S.D. & $P$ \\
\hline \multicolumn{13}{|l|}{ Functioning scales ${ }^{a}$} \\
\hline Physical functioning & 20 & 79.3 & 17.3 & -2.0 & 17.7 & 0.619 & 18 & 80.6 & 18.2 & -3.1 & 12.2 & 0.290 \\
\hline Role functioning & 20 & 74.2 & 27.8 & -16.7 & 44.3 & 0.109 & 18 & 63.0 & 29.5 & -1.9 & 26.1 & 0.767 \\
\hline Cognitive functioning & 20 & 85.8 & 20.4 & -1.7 & 20.2 & 0.716 & 18 & 86.1 & 14.3 & -3.7 & 13.5 & 0.260 \\
\hline Emotional functioning & 20 & 74.2 & 15.7 & 0.8 & 18.7 & 0.844 & 18 & 75.9 & 19.8 & -4.6 & 12.9 & 0.145 \\
\hline Social functioning & 20 & 83.3 & 18.7 & -7.5 & 39.5 & 0.407 & 18 & 83.3 & 26.2 & -0.9 & 18.5 & 0.834 \\
\hline \multicolumn{13}{|l|}{ Overall rating of quality of life ${ }^{a}$} \\
\hline Global quality of life & 20 & 65.0 & 18.5 & -5.0 & 22.2 & 0.326 & 18 & 64.4 & 21.4 & -0.9 & 13.1 & 0.767 \\
\hline \multicolumn{13}{|l|}{ Symptoms: scales and single items ${ }^{b}$} \\
\hline Fatigue & 20 & 32.2 & 24.2 & 7.2 & 23.2 & 0.179 & 18 & 35.8 & 20.0 & 3.1 & 19.0 & 0.500 \\
\hline Nausea and vomiting & 20 & 4.2 & 9.2 & 5.0 & 15.4 & 0.163 & 18 & 7.4 & 11.7 & 2.8 & 10.3 & 0.269 \\
\hline Pain & 20 & 19.2 & 23.7 & -1.7 & 28.6 & 0.797 & 18 & 13.0 & 19.4 & 7.4 & 21.6 & 0.163 \\
\hline Dyspnoea & 20 & 23.3 & 28.8 & 1.7 & 20.2 & 0.716 & 18 & 20.4 & 23.3 & 0 & 19.8 & 1.00 \\
\hline Insomnia & 20 & 18.3 & 25.3 & -3.3 & 32.3 & 0.649 & 18 & 9.3 & 19.2 & 9.3 & 27.5 & 0.172 \\
\hline Appetite loss & 20 & 13.3 & 22.7 & 6.7 & 27.8 & 0.297 & 18 & 20.4 & 28.3 & 11.1 & 25.6 & 0.083 \\
\hline Constipation & 20 & 3.3 & 14.9 & 5.0 & 12.2 & 0.083 & 18 & 3.7 & 10.8 & 0 & 11.4 & 1.00 \\
\hline Diarrhoea & 19 & 33.3 & 29.4 & -5.3 & 22.9 & 0.331 & 17 & 19.6 & 23.7 & 7.8 & 22.1 & 0.163 \\
\hline Financial impact & 20 & 1.7 & 7.5 & -1.7 & 7.5 & 0.330 & 18 & 0 & 0 & 1.9 & 7.9 & 0.331 \\
\hline Flushes & 20 & 48.3 & 27.5 & -15.0 & 27.5 & 0.025 & 18 & 27.8 & 23.6 & 1.9 & 26.7 & 0.772 \\
\hline Fevers & 20 & 10.0 & 21.9 & -8.3 & 23.9 & 0.135 & 18 & 1.9 & 7.9 & 0 & 11.4 & 1.00 \\
\hline Muscle pain & 20 & 26.7 & 29.8 & -1.7 & 29.6 & 0.804 & 18 & 22.2 & 19.8 & 7.4 & 29.3 & 0.298 \\
\hline Abdominal pain & 18 & 14.8 & 20.5 & 9.3 & 27.5 & 0.172 & 17 & 23.5 & 30.7 & -9.8 & 28.3 & 0.172 \\
\hline $\begin{array}{l}\text { Worries about disease progression } \\
\text { Depression }^{\mathrm{b}}\end{array}$ & 20 & 60.0 & 23.2 & -18.3 & 27.5 & 0.008 & 18 & 37.0 & 25.3 & -3.7 & 19.4 & 0.430 \\
\hline CES-D & 19 & 11.3 & 7.0 & 1.7 & 6.7 & 0.272 & 18 & 12.0 & 7.7 & 0.5 & 5.8 & 0.704 \\
\hline
\end{tabular}

$\mathrm{T}_{0}-\mathrm{T}_{8}$; interval week 0-8, $\mathrm{T}_{8}-\mathrm{T}_{21}$; interval week 8-21.

a Higher score indicates better functioning: a negative change score is a deteroriation in functioning, a positive change score an improvement.

${ }^{b}$ Higher score indicates more symptoms: a positive change score is an increase in symptoms, a negative change score a decrease.

with hepatitis B (Itri et al. 1989). This was confirmed in a more recent paper describing patients with hepatitis $\mathrm{C}$ treated with IFN (Hou et al. 2000). In a study among 327 carcinoid patients, those with a high titre of antibodies lost the response to treatment, but this phenomenon occurred late in the disease after a median of 25 months (Oberg \& Alm 1997).

The second step in our treatment protocol was the administration of unlabelled MIBG, the cytotoxic effect of which is related to inhibition of mitochondrial respiration. In a previous series of 20 carcinoid patients, three cycles of unlabelled MIBG resulted in a good palliative effect in 60\% (Taal et al. 1996). Apart from combining the two active treatment modalities, we hypothesised that IFN- $\alpha$ might induce differentiation of tumour cells, leading to facilitation of MIBG transport and uptake, and hence improved clinical results. This was based on the in vitro results of IFN- $\alpha$ in a neuroblastoma cell line (Montaldo et al. 1992). As differentiation might occur with IFN- $\alpha$ as well after some time in vivo (Oberg 1992), we choose a period of 2 months of treatment with IFN- $\alpha$.

A favourable effect upon biodistribution (>10\% increase) at the ${ }^{131}$ I-MIBG scintigraphy was seen in only $19 \%$ of the patients. Overall, IFN did not affect the MIBG uptake in carcinoid patients significantly. This might be explained by a different effect of IFN- $\alpha$ compared with IFN- $\gamma$ as used in the cell-line model, or a different reaction of the carcinoid tumour cells compared with the neuroblastoma cells. Another explanation that the duration of treatment ( 8 weeks) might have been too short to induce cell differentiation seems unlikely. However, significant increase in biodistribution and tumour uptake was induced by unlabelled MIBG given directly prior to the ${ }^{131} \mathrm{I}-\mathrm{MIBG}$ scintigraphy in $83 \%$.

The combination of MIBG and IFN was well tolerated, and in only one patient was a transient rise in 
blood pressure seen as an adverse effect. Unfortunately, the addition of MIBG to IFN resulted in only one more response, leading to an overall response rate of $39 \%$.

Quality of life measurements of the five functioning scales as well as global health showed no significant improvement. However, among the single-item scores, flushes and worries about disease progression improved significantly. The only other study dealing with quality of life evaluation in relation to treatment is by Wymenga et al. (1999), in which the somatostatin analogue lanreotide resulted in an early but temporary improvement of emotional and cognitive functioning, as well as the global health scale. As serotonin is involved in the aetiology of depression and depression is one of the possible sideeffects of IFN, we also applied questionnaires to measure the symptoms of depression. However, clinically important depression was not seen before the start and did not develop during the treatment. These findings are in line with the report of 24 carcinoid patients in Sweden in whom a low level of anxiety and depression was found during a year of follow-up (Larsson et al. 2001).

To study the symptoms in a different manner, we also divided the patients into two groups based on the predominant symptoms. Twenty-nine percent of patients with the carcinoid syndrome mentioned an additional improvement with unlabelled MIBG, and $75 \%$ of cases of systemic symptoms such as fever, anorexia and fatigue. Compared with a $60 \%$ response rate as a single agent (Taal et al. 1996), the combination of unlabelled MIBG with IFN is probably not more effective.

The final step in the protocol consisted of radioactive MIBG as an adjunct to IFN treatment in cases of positive scintigraphy, to provide a local irradiation dose. In the present study, 14 patients received at least one cycle resulting in one PR. ${ }^{131} \mathrm{I}-\mathrm{MIBG}$ has been shown to induce long-term palliative effect in $60 \%$ of patients with metastatic carcinoid tumours described earlier by our group (Taal et al. 1996, 2000). Mukherjee et al. (2001) reported similar favourable results with a subjective response in 11 out of $18(73 \%)$ patients. In another study including several types of neuroendocrine tumours (Pathirana et al. 2001), a subjective response rate of $83 \%$ was reported in the subgroup of ten patients with carcinoid tumours, following only one cycle of ${ }^{131}$ I-MIBG.

The side-effects of radioactive MIBG were not increased by the concomitant use of IFN and were still limited to nausea and vomiting in a small minority of patients.

The treatment with radiolabelled MIBG is only given to patients with a positive MIBG scintigraphy. To be able to select more patients for this treatment, we could improve results by pretreatment with unlabelled MIBG as described earlier (Taal et al. 2000). In the present study, long-term pretreatment with IFN did not show such a favourable effect.

In conclusion, IFN- $\alpha$ results are comparable with those described in the literature. Although an increased uptake of ${ }^{131}$ I-MIBG was seen in $19 \%$, the increased clinical response rate to combined treatment with both unlabelled and ${ }^{131}$ I-labelled MIBG was disappointing and restricted to patients with the carcinoid syndrome or other systemic symptoms.

\section{Acknowledgements}

We would like to thank J H Beijnen, Department of Pharmacy and Pharmacology, Slotervaart Hospital, Amsterdam, The Netherlands for the preparation of unlabelled MIBG and R Kröger, radiologist, for revision of the CT scans.

\section{References}

Caplin ME, Buscombe JR, Hilson AJ, Jones AL, Watkinson AF \& Burroughs AK 1998 Carcinoid tumour. Lancet 352 799-805.

Detjen KM, Welzel M, Farwig K, Brembeck FH, Kaiser A, Riecken EO, Wiedenmann B \& Rosewicz S 2000 Molecular mechanism of interferon alfa-mediated growth inhibition in human neuroendocrine tumor cells. Gastroenterology 118 735-748.

Eissele R, Anlauf M, Schafer MK, Eiden LE, Arnold R \& Weihe E 1999 Expression of vesicular monoamine transporters in endocrine hyperplasia and endocrine tumors of the oxyntic stomach. Digestion 60 428-439.

Geerlings SW, Beekman AT, Deeg DJ \& Van Tilburg W 2000 Physical health and the onset and persistence of depression in older adults: an eight-wave prospective community-based study. Psychological Medicine 30 369-380.

Harris AG \& Redfern JS 1995 Octreotide treatment of carcinoid syndrome: analysis of published dose-titration data. Alimentary Pharmacology and Therapeutics 9 387-394.

Hoefnagel CA 1991 Radionuclide therapy revisited. European Journal of Nuclear Medicine 18 408-431.

Hoefnagel CA, Taal BG, Sivro F, Boot H \& Valdes Olmos RA 2000 Enhancement of ${ }^{131}$ I-MIBG uptake in carcinoid tumours by administration of unlabelled MIBG. Nuclear Medicine Communications 21 755-761.

Hou C, Chuang WL, Yu ML, Dai CY, Chen SC, Lin ZY, Hsieh MY, Wang LY, Tsai JF \& Chang WY 2000 Incidence and associated factors of neutralizing anti-interferon antibodies among chronic hepatitis $\mathrm{C}$ patients treated with interferon in Taiwan. Scandinavian Journal of Gastroenterology 35 1288-1293.

Itri LM, Sherman MI, Palleroni AV, Evans LM, Tran LL, Campion M \& Chizzonite R 1989 Incidence and clinical significance of neutralizing antibodies in patients receiving 
recombinant interferon-alpha 2a. Journal of Interferon Research 9 (Suppl 1) S9-S15.

Kolby L, Bernhardt P, Levin-Jakobsen AM, Johanson V, Wangberg B, Ahlman H, Forssell-Aronsson E \& Nilsson O 2003 Uptake of meta-iodobenzylguanidine in neuroendocrine tumours is mediated by vesicular monoamine transporters. British Journal of Cancer 89 1383-1388.

Kuin A, Smets L, Volk T, Paans A, Adams G, Atema A, Jahde E, Maas A, Rajewsky MF \& Visser G 1994 Reduction of intratumoral $\mathrm{pH}$ by the mitochondrial inhibitor m-iodobenzylguanidine and moderate hyperglycemia. Cancer Research 54 3785-3792.

Langendijk JA, Aaronson NK, de Jong JM, ten Velde GP, Muller MJ, Lamers RJ, Slotman BJ \& Wouters EF 2001 Prospective study on quality of life before and after radical radiotherapy in non-small-cell lung cancer. Journal of Clinical Oncology 19 2123-2133.

Larsson G, Sjoden PO, Oberg K, Eriksson B \& von Essen L 2001 Health-related quality of life, anxiety and depression in patients with midgut carcinoid tumours. Acta Oncologica 40 825-831.

Moertel CG, Rubin J \& Kvols LK 1989 Therapy of metastatic carcinoid tumor and the malignant carcinoid syndrome with recombinant leukocyte A interferon. Journal of Clinical Oncology 7 865-868.

Montaldo PG, Carbone R, Ponzoni M \& Cornaglia-Ferraris P $1992 \gamma$-Interferon increases metaiodobenzylguanidine incorporation and retention in human neuroblastoma cells. Cancer Research 52 4960-4964.

Mukherjee JJ, Kaltsas GA, Islam N, Plowman PN, Foley R, Hikmat J, Britton KE, Jenkins PJ, Chew SL, Monson JP, Besser GM \& Grossman AB 2001 Treatment of metastatic carcinoid tumours, phaeochromocytoma, paraganglioma and medullary carcinoma of the thyroid with $\left({ }^{131}\right)$ I-metaiodobenzylguanidine $\left[\left({ }^{131}\right) \mathrm{I}-\mathrm{mIBG}\right]$. Clinical Endocrinology 55 47-60.

Oberg K 1992 The action of interferon alpha on human carcinoid tumours. Seminars in Cancer Biology 3 35-41.

Oberg K 1994 Treatment of neuroendocrine tumors. Cancer Treatment Reviews 20 331-355.

Oberg K \& Alm G 1997 The incidence and clinical significance of antibodies to interferon-a in patients with solid tumors. Biotherapy 10 1-5.
Oberg K, Eriksson B \& Janson ET 1994 The clinical use of interferons in the management of neuroendocrine gastroenteropancreatic tumors. Annals of the New York Academy of Sciences 33 471-478.

Pathirana AA, Vinjamuri S, Byrne C, Ghaneh P, Vora J \& Poston GJ $2001\left({ }^{131}\right)$ I-MIBG radionuclide therapy is safe and cost-effective in the control of symptoms of the carcinoid syndrome. European Journal of Surgical Oncology 27 404-408.

Quaedvlieg PF, Visser O, Lamers CB, Janssen-Heijen ML \& Taal BG 2001 Epidemiology and survival in patients with carcinoid disease in The Netherlands. An epidemiological study with 2391 patients. Annals of Oncology 12 1295-1300.

Radloff LS 1977 The CES-D scale: A self-report depression scale for research in the general population. Applied Psychological Measurement 1 385-401.

Sarna L, Padilla G, Holmes C, Tashkin D, Brecht ML \& Evangelista L 2002 Quality of life of long-term survivors of non-small-cell lung cancer. Journal of Clinical Oncology 20 2920-2929.

Schellekens H, Ryff JC \& van der Meide PH 1997 Assays for antibodies to human interferon-alpha: the need for standardization. Journal of Interferon and Cytokine Research 17 (Suppl 1) S5-S8.

Taal BG, Hoefnagel CA, Valdes Olmos RA, Boot H \& Beijnen JH 1996 Palliative effect of metaiodobenzylguanidine in metastatic carcinoid tumors. Journal of Clinical Oncology 14 $1829-1838$

Taal BG, Hoefnagel C, Boot H, Valdes OR \& Rutgers M 2000 Improved effect of ${ }^{131}$ I-MIBG treatment by predosing with non-radiolabeled MIBG in carcinoid patients, and studies in xenografted mice. Annals of Oncology 11 1437-1443.

Wymenga AN, Eriksson B, Salmela PI, Jacobsen MB, Van Cutsem EJ, Fiasse RH, Valimaki MJ, Renstrup J, de Vries EG \& Oberg KE 1999 Efficacy and safety of prolongedrelease lanreotide in patients with gastrointestinal neuroendocrine tumors and hormone-related symptoms. Journal of Clinical Oncology 17 1111-1117.

Zuetenhorst H, Taal BG, Boot H, Valdes OR \& Hoefnagel C 1999 Long-term palliation in metastatic carcinoid tumours with various applications of meta-iodobenzylguanidin (MIBG): pharmacological MIBG, ${ }^{131}$ I-labelled MIBG and the combination. European Journal of Gastroenterology and Hepatology 11 1157-1164. 